\title{
Field-Assisted Nanopatterning
}

\section{Jun-Fu Liu and Glen P. Miller}

Department of Chemistry and Materials Science Program

University of New Hampshire

Durham, NH 03824

glen.miller@unh.edu

Jun-Fu Liu and Glen P. Miller*

University of New Hampshire 


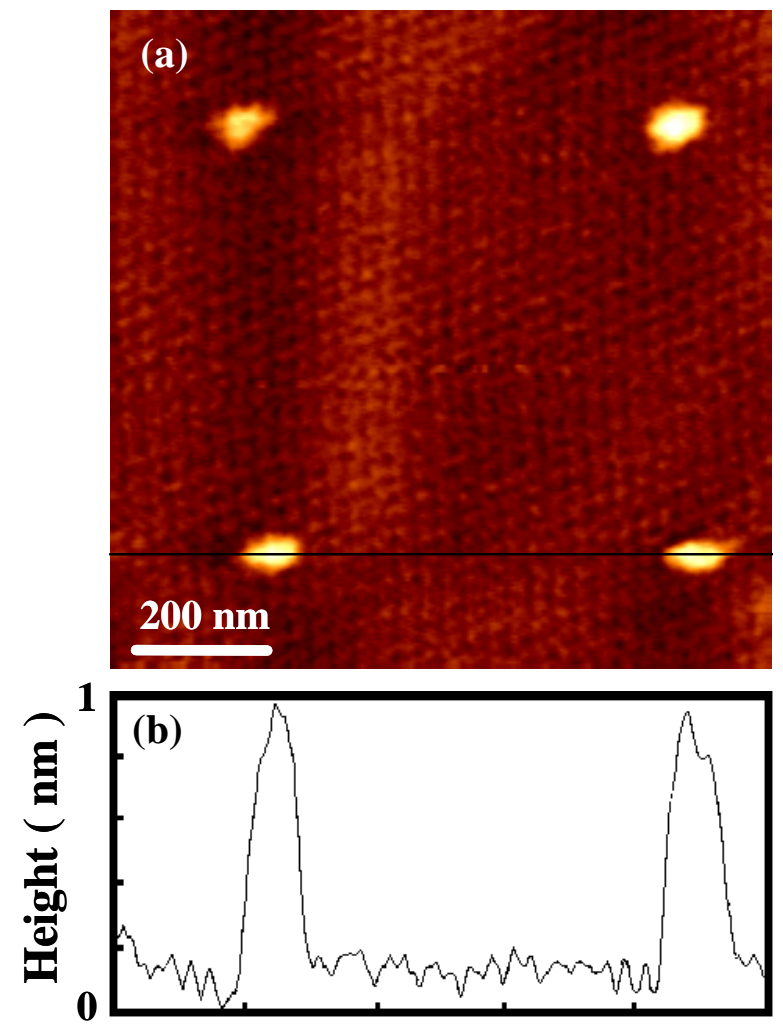

Field-assisted nanopatterning of [60]fullerene dots on HOPG

(a) height image, (b) cursor plot. The nanodots were fabricated using a tip bias of $-4 \mathrm{~V}$ and a tip hold time of $500 \mathrm{~ms}$. 

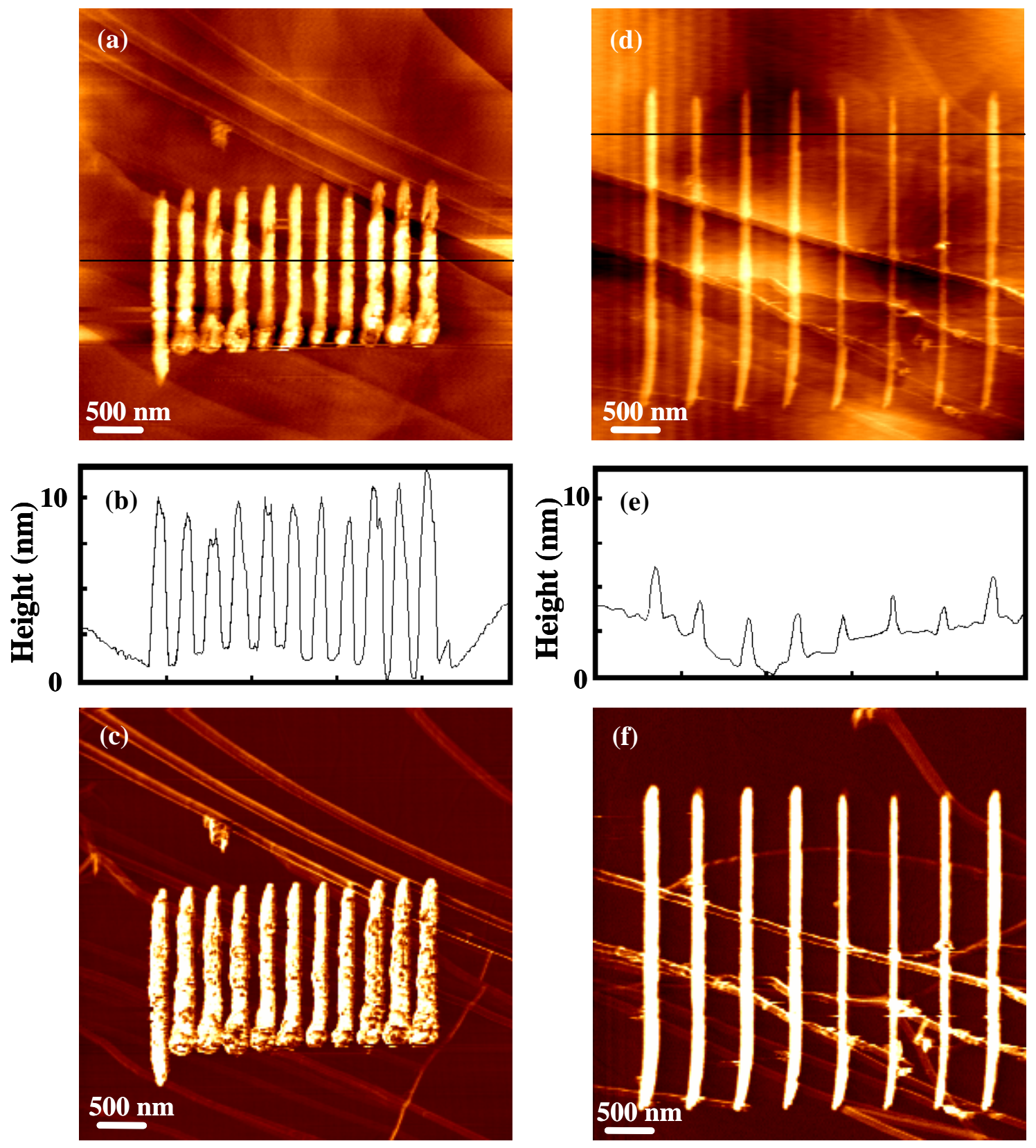

Field-assisted nanopatterning of [60]fullerene lines on HOPG

(a, d) height images; (b, e) cursor plots; (c, f) friction images. The brighter areas in (c) and (f) indicate higher friction. $(\mathrm{a}-\mathrm{c})$ fabricated at a tip bias of $-6 \mathrm{~V}$ and a fabrication speed of 100 $\mathrm{nm} / \mathrm{s},(\mathrm{d}-\mathrm{f})$ fabricated at a tip bias of $-6 \mathrm{~V}$ and a fabrication speed of $500 \mathrm{~nm} / \mathrm{s}$. 

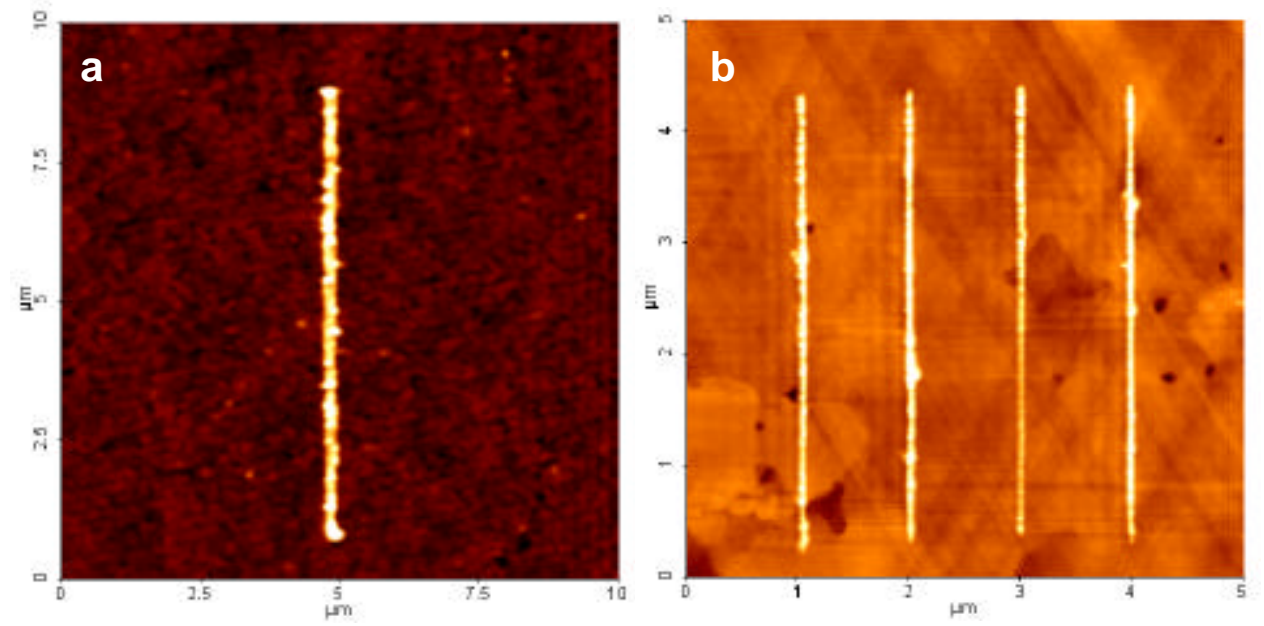

Field-assisted nanopatterning of [60]fullerene lines on ITO and 1-octadecanethiol coated Au

(a) ITO substrate; (b) 1-octadecanethiol coated Au(111) substrate.

Both (a) and (b) are height images. The nanolines were fabricated using tip bias of $-8 \mathrm{~V}$ (a) and $-10 \mathrm{~V}$ (b) and a fabrication speed of $100 \mathrm{~nm} / \mathrm{s}$. 

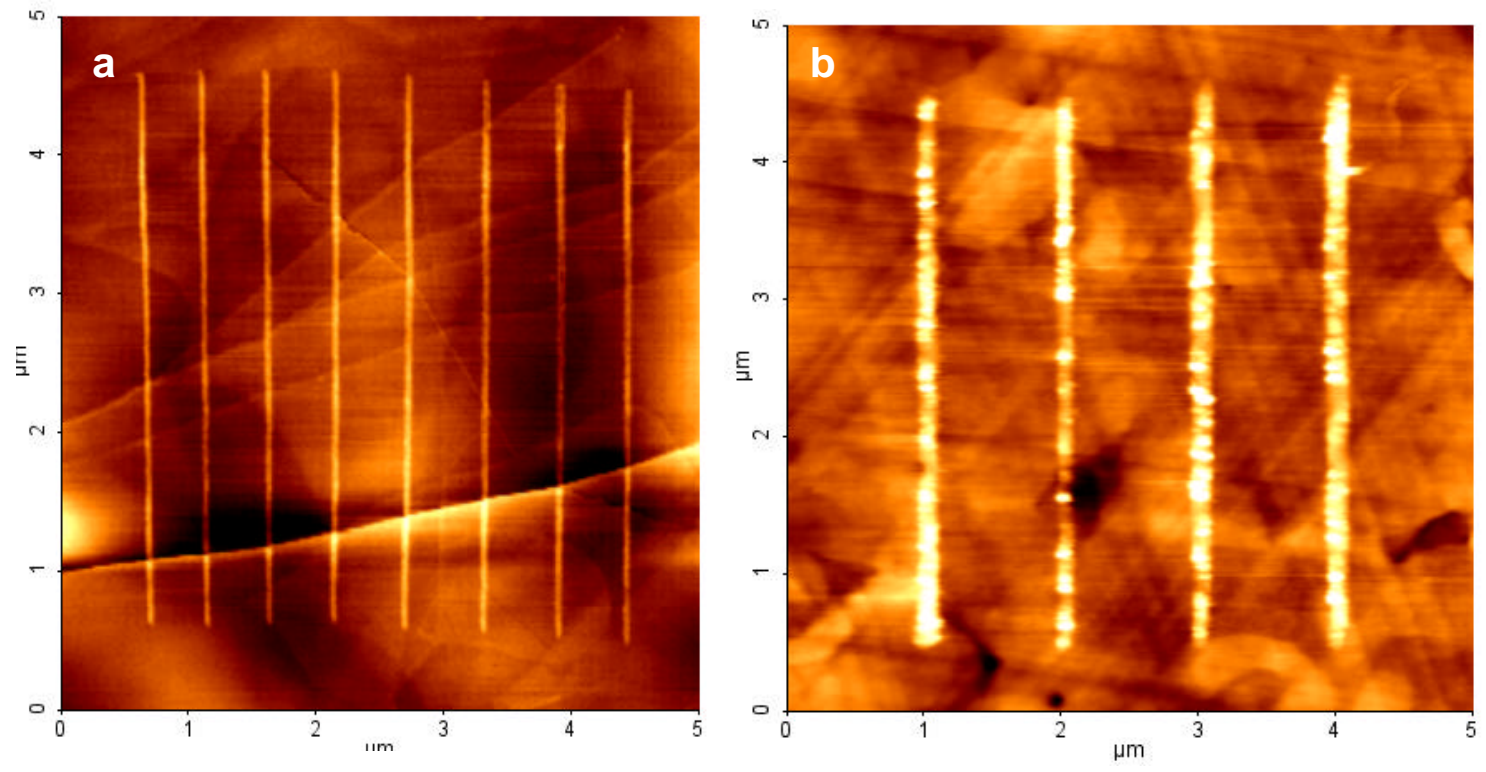

Field-assisted nanopatterning of naphthalene lines on HOPG and octadecanethiol/Au

(a) HOPG substrate; (b) octadecanethiol coated Au(111) substrate.

Both (a) and (b) are height images. The naphthalene lines were fabricated using a tip bias of -9 $\mathrm{V}$ (a) and $-10 \mathrm{~V}$ (b) and a fabrication speed of $100 \mathrm{~nm} / \mathrm{s}$. 

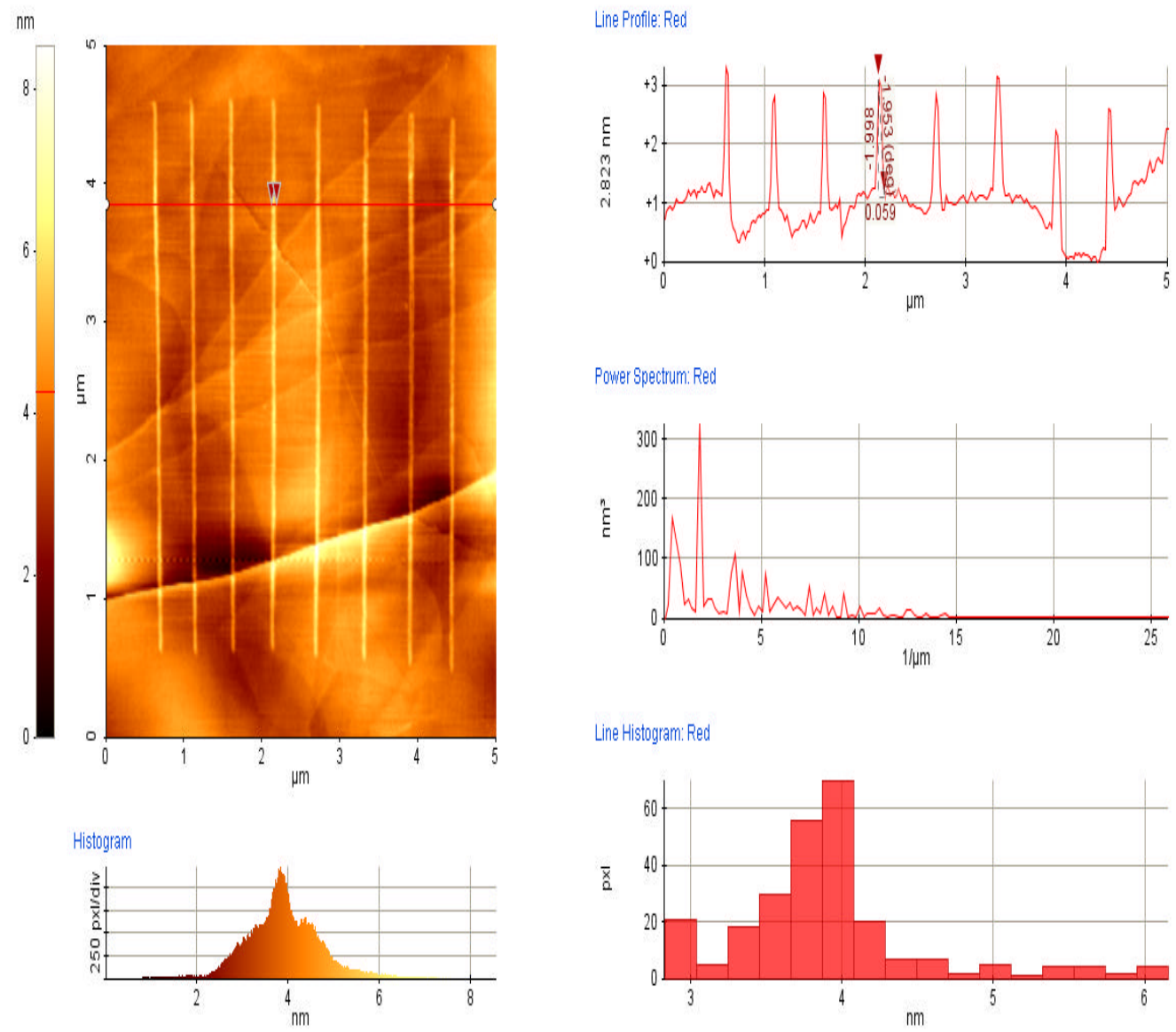

Line Histogram: Red

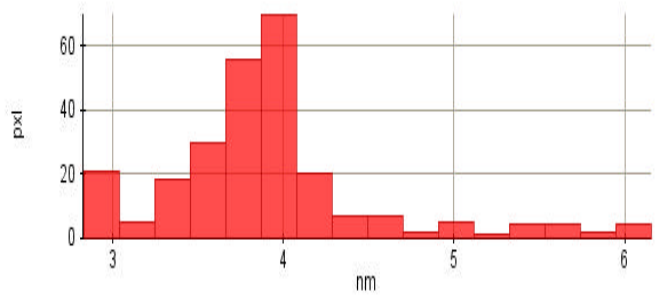

Statistics

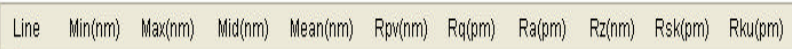

$\begin{array}{lllllllllll}\text { マRed } & 2.823 & 6.145 & 4.484 & 3.895 & 3.322 & 616.933 & 407.076 & 1.057 & .31 .336 & 137.643\end{array}$

Field-assisted nanopatterning of naphthalene on HOPG

A tip bias value of $-9 \mathrm{~V}$ and a tip speed of $100 \mathrm{~nm} / \mathrm{s}$ was utilized.

Jun-Fu Liu and Glen P. Miller*

University of New Hampshire 

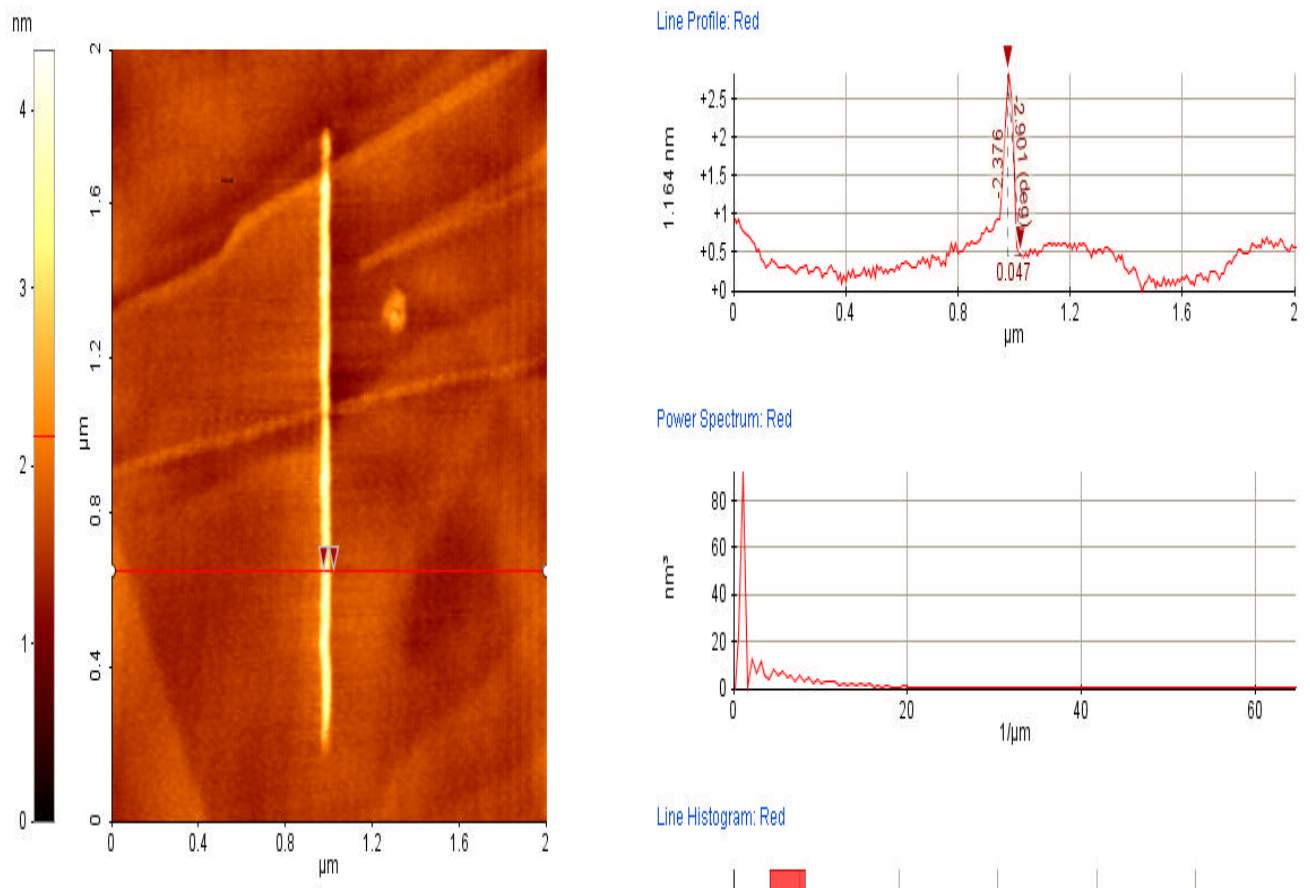

Power Spectrum: Red

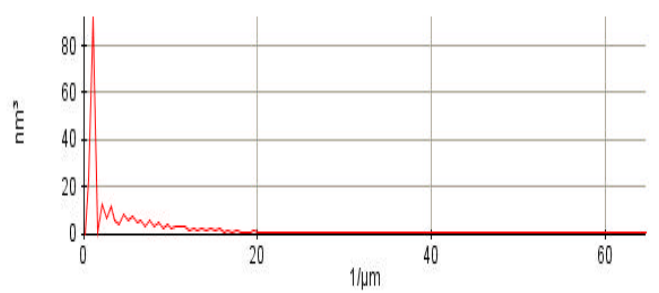

Line Histogram: Red

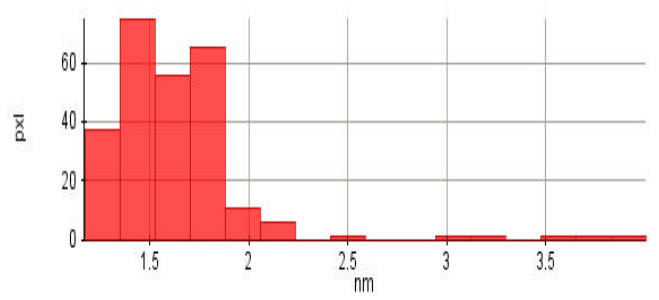

Statistics

Line Min(nm) Max(nm) Mid(nm) Mean(nm) Rpv(nm) Ra(pm) Ra(pm) Rz(nm) Rsk(nm) Rku(pm)

$\begin{array}{lllllllllll}\nabla \text { Red } & 1.164 & 4.000 & 2.582 & 1.614 & 2.836 & 346.661 & 213.705 & 1.519 & -87.900 & 535.935\end{array}$

Field-assisted nanopatterning of naphthalene on HOPG

A tip bias value of $-8 \mathrm{~V}$ and a tip speed of $500 \mathrm{~nm} / \mathrm{s}$ was utilized. 

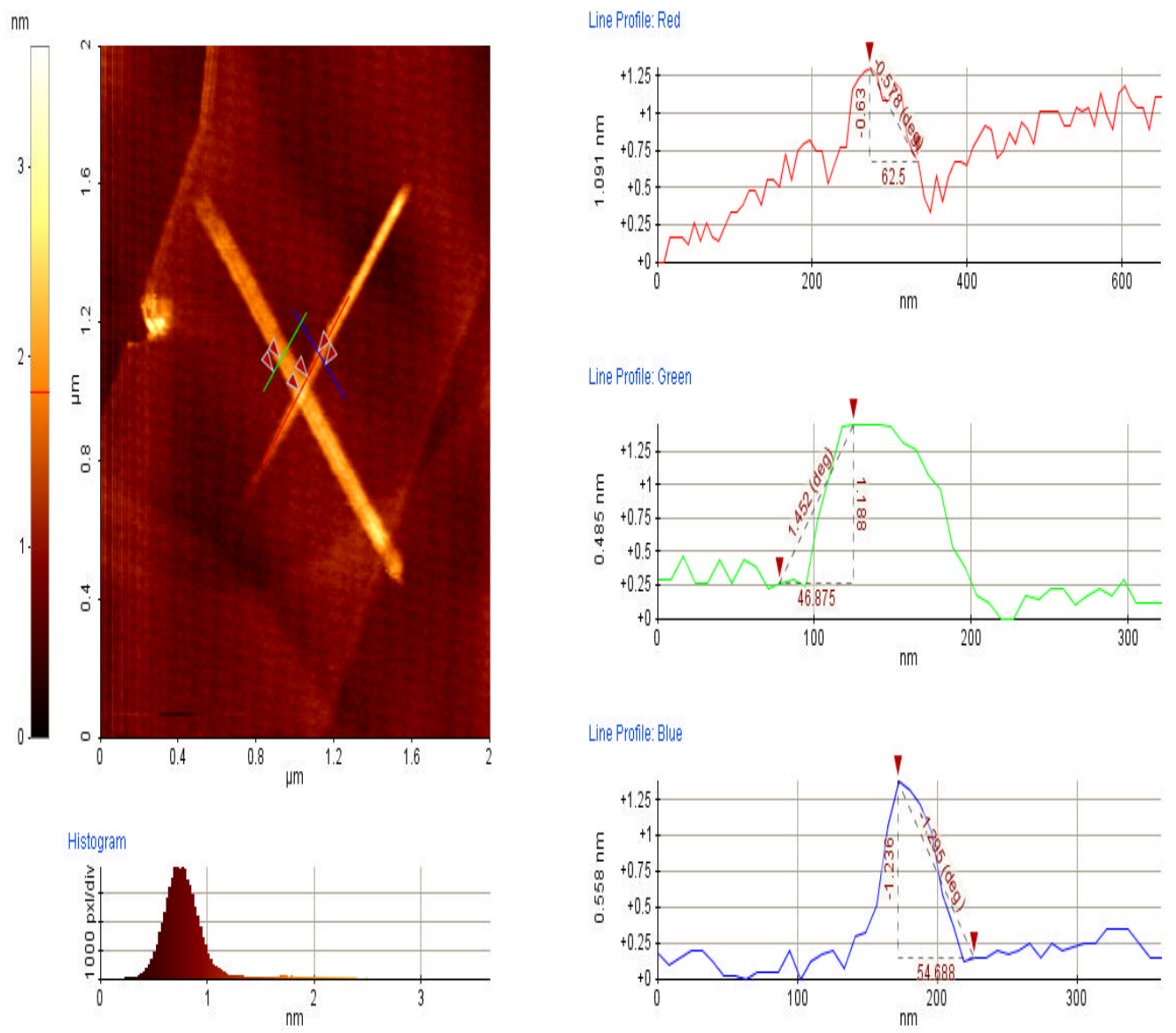

\begin{tabular}{|c|c|c|c|c|c|c|c|c|c|c|}
\hline Line & Min(ninm) & Max(nm) & Mid(n) & Mean(nm) & Rppy(nm) & $\operatorname{Rig}(\mathrm{pm})$ & Ral(pm) & $\operatorname{Rz}(\mathrm{pm})$ & Rsk(pm) & Rku(pm) \\
\hline IRed & 1.091 & 2.400 & 1.745 & 1.825 & 1.309 & 336.783 & 278.951 & 92.060 & 9.651 & 53.856 \\
\hline Green & 0.485 & 1.939 & 1.212 & 0.996 & 1.455 & 471.478 & 3939.926 & 490.985 & .25 .584 & 61.641 \\
\hline IBlue & 0.558 & 1.939 & 1.248 & 0.858 & 1.382 & 337.478 & 224.819 & 616.163 & .50 .500 & 152.932 \\
\hline
\end{tabular}

Field-assisted nanopatterning of criss-crossing naphthalene lines on HOPG

A tip bias value of $-6 \mathrm{~V}$ and a tip speed of $500 \mathrm{~nm} / \mathrm{s}$ was utilized. 


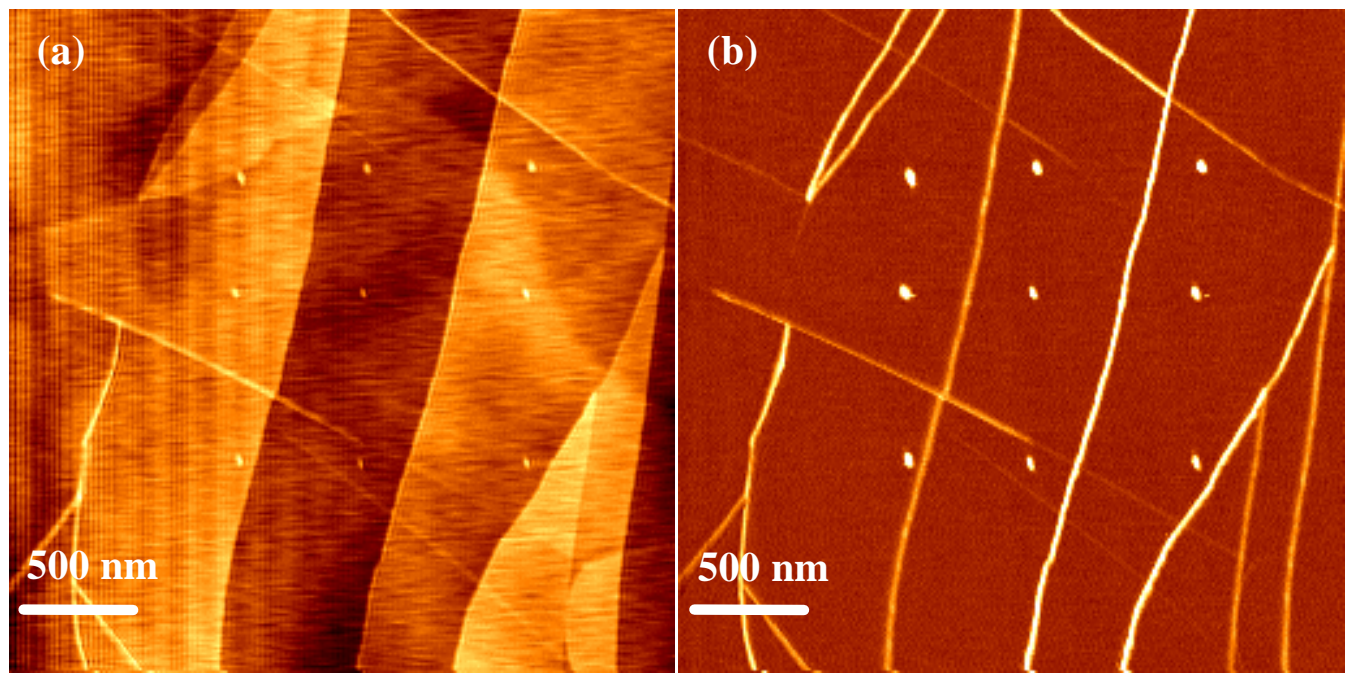

Field-assisted nanopatterning of N-methylpyrrole nanodots on HOPG

(a) height image; (b) friction image. The brighter areas in (b) indicate higher friction. The nanodots were fabricated using a tip bias of $-6 \mathrm{~V}$ and a tip hold time of $500 \mathrm{~ms}$. 

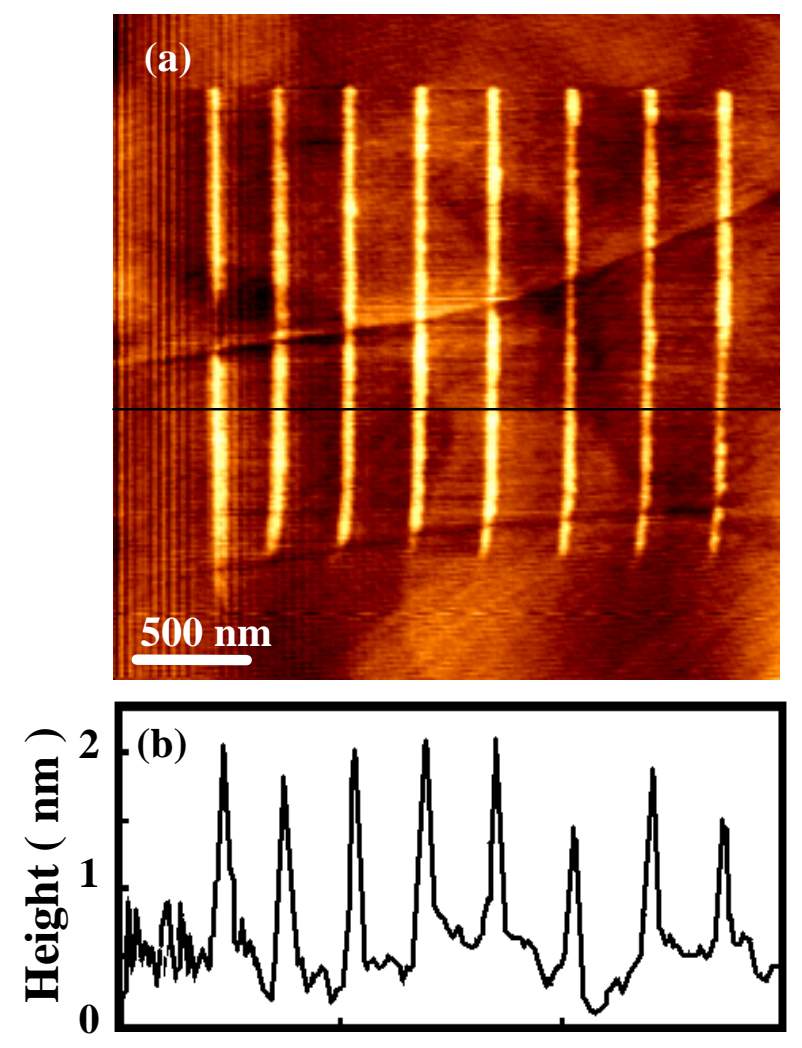

Field-assisted nanopatterning of N-methylpyrrole lines on HOPG

(a) height image; (b) cursor plot. The lines were fabricated at tip bias $-9 \mathrm{~V}$ and a fabrication speed of $500 \mathrm{~nm} / \mathrm{s}$. 

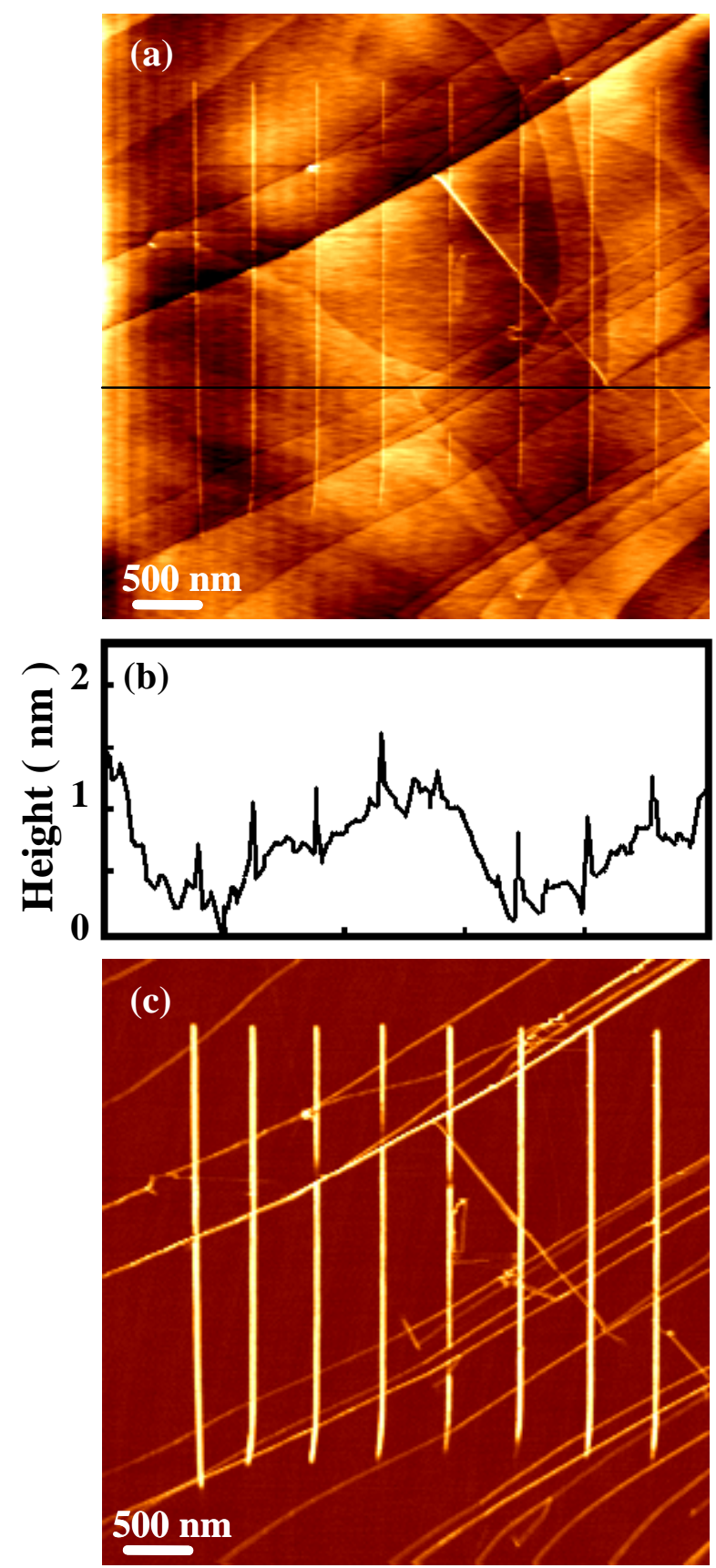

Field-assisted nanopatterning of meso-tetraphenylporphrin lines on HOPG

(a) height image; (b) cursor plot; (c) friction images. The brighter areas in (c) indicate higher friction. The lines were fabricated using a tip bias of $-7 \mathrm{~V}$ and a fabrication speed of $500 \mathrm{~nm} / \mathrm{s}$. 


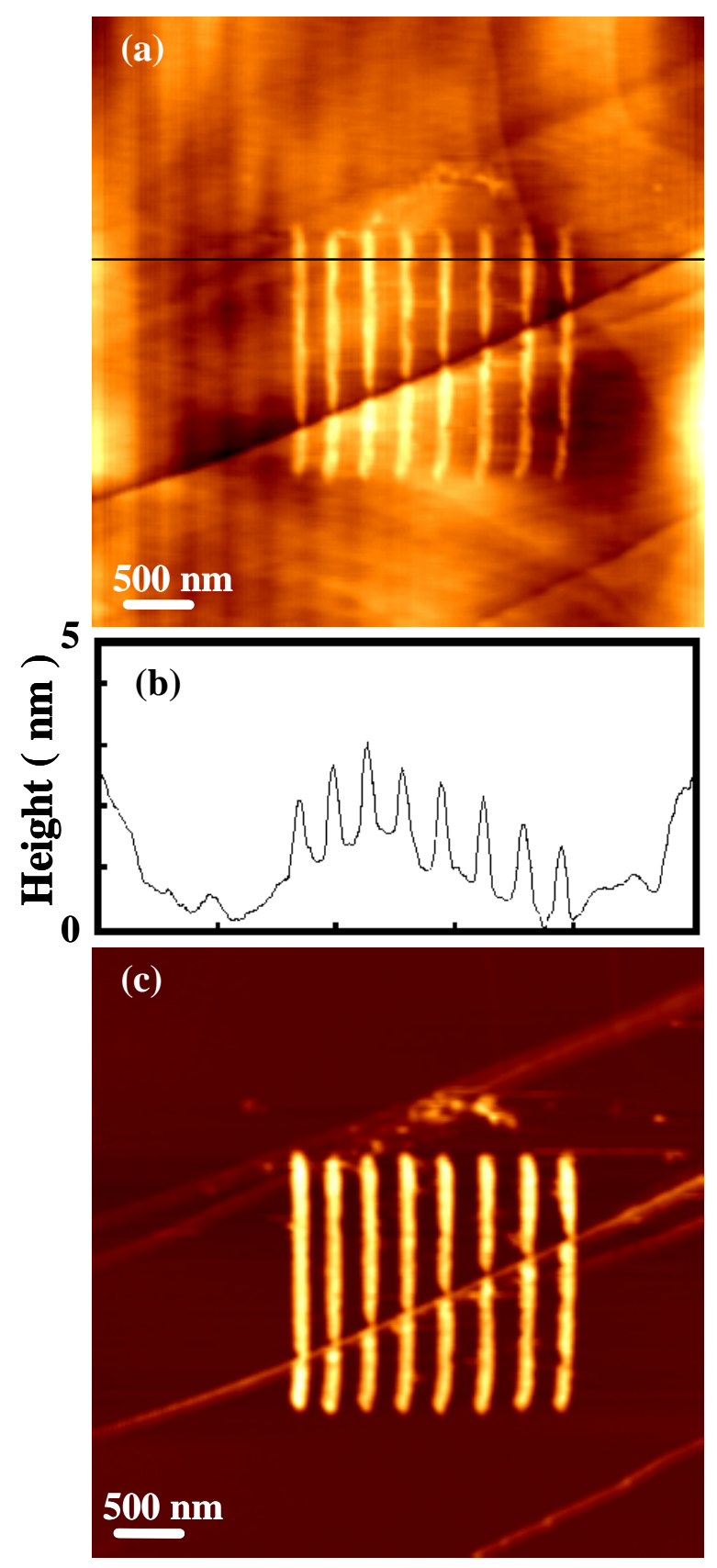

Field-assisted nanopatterning of poly-3-octylthiophene-2,5-diyl lines on HOPG

(a) height image; (b) cursor plot; (c) friction image. The brighter areas in (c) indicate higher friction. The lines were fabricated at tip bias $-7 \mathrm{~V}$ and a fabrication speed of $100 \mathrm{~nm} / \mathrm{s}$. 

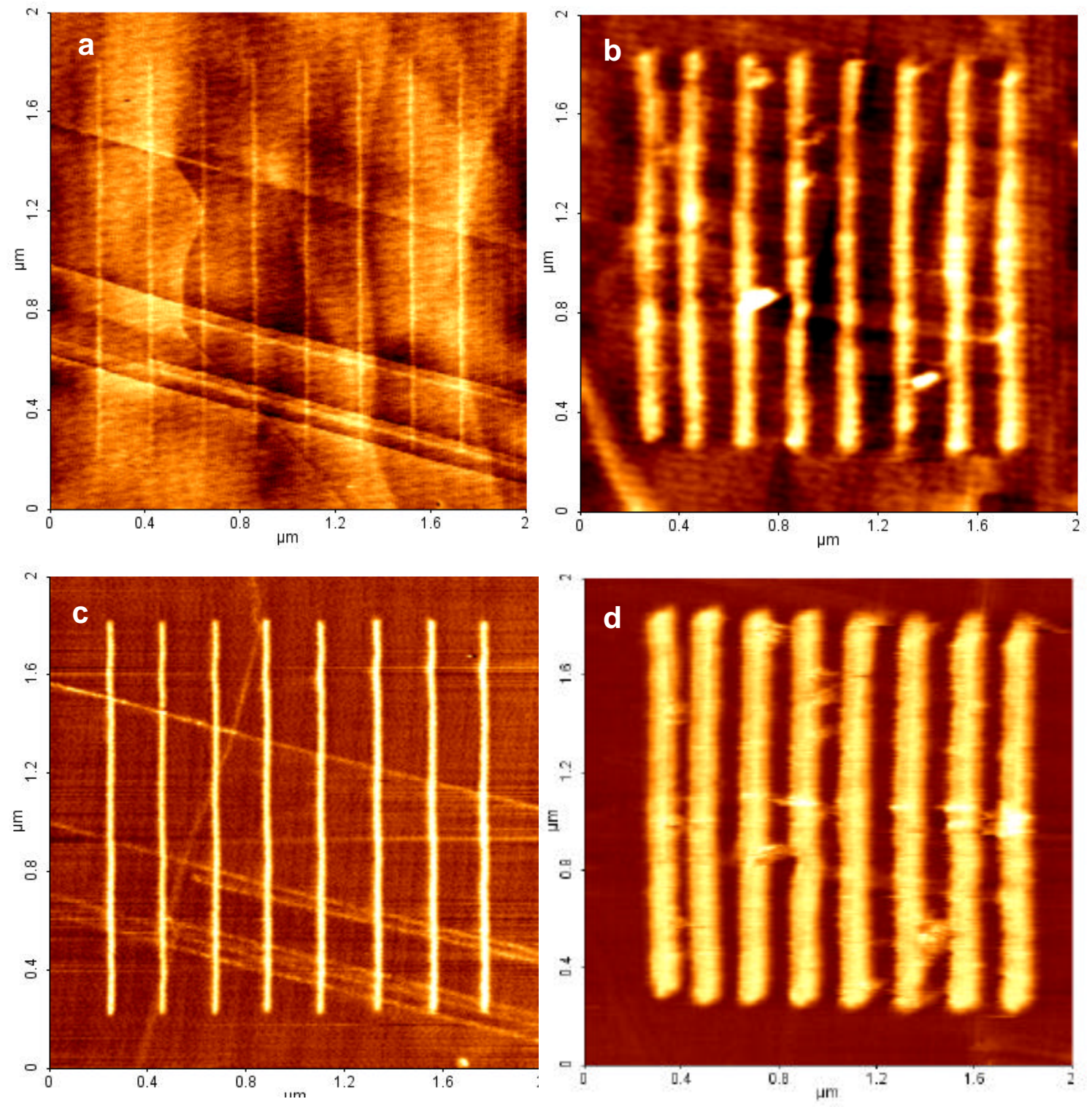

Field-assisted nanopatterning of polyaniline lines on HOPG

(a, b) height images, (c, d) friction images. The brighter areas in (c,d) indicate higher friction. The lines were fabricated at a tip bias of $-8 \mathrm{~V}$ and fabrication speeds of $500 \mathrm{~nm} / \mathrm{s}(\mathrm{a}, \mathrm{c})$ and $100 \mathrm{~nm} / \mathrm{s}(\mathrm{b}, \mathrm{d})$ 